\title{
Bipolarity and Multipolarity in Aggregation Structures
}

\author{
Guy De Tré \\ Dept. of Telecommunications and \\ Information Processing, \\ Ghent University \\ Sint-Pietersnieuwstraat 41, \\ B-9000 Ghent, Belgium \\ Email: Guy.DeTre@UGent.be
}

\author{
Jozo J. Dujmović \\ Dept. of Computer Science \\ San Francisco State University \\ 1600 Holloway Avenue, \\ San Francisco, CA 94132, USA \\ Email: jozo@sfsu.edu
}

\author{
Sławomir Zadrożny \\ Systems Research Institute \\ Polish Academy of Science \\ Newalska 6, \\ 01-447 Warsaw, Poland \\ Email: zadrozny@ibspan.waw.pl
}

\begin{abstract}
In recent years, there has been an increasing interest in handling (heterogeneous) bipolarity in order to deal more adequately with user preferences in information management. Different approaches have been presented. With respect to criteria specification in either flexible database querying or decision support, a main distinction can be made between bipolarity that is specified inside a single criterion and bipolarity that is specified among multiple criteria. Consider criteria that are defined over the values of a given domain. With bipolarity inside a criterion specification, a user might for example state which domain values she likes (positive pole) and which domain values she doesn't like (negative pole). Herewith, both poles do not have to be the complement of each other. With bipolarity among multiple criteria, in general two poles of criteria are given different semantics and handled in a different way. In this paper we survey different forms of bipolarity among multiple criteria from the standpoint of aggregation and try to classify them into two groups: bipolarity based on positive (desirable) and negative (undesirable) criteria and bipolarity based on nonuniform inputdependent annihilators. Moreover, we present canonical forms of aggregators of the two groups.
\end{abstract}

\section{INTRODUCTION}

In the context of information management the term bipolarity is, among others, used to refer to different poles of values or different poles of criteria which have to be interpreted and handled in a different way.

When different poles of values are considered, one often distinguishes between positive values, i.e., values which are possible, satisfactory, permitted, desired or acceptable, and negative values, i.e., values which are impossible, unsatisfactory, not permitted, rejected, undesired or unacceptable. A typical application is the specification of query preferences where a user specifies for a given criterion which domain values are acceptable (to a certain extent) and which are not. For example, consider the specification of user preferences in the context of selecting the color of a car. For a given user, the positive pole of values might consist of black, dark blue, and dark brown, whereas the negative pole consists of white, yellow and purple. For other colors, e.g., red, there is some indifference. These colors are neither in the positive, nor in the negative pole, what illustrates the heterogeneous character of the bipolarity. We refer to bipolarity that relates to a single domain of values as bipolarity that is specified inside a single criterion (cf. also [21], [23] where we refer to it as bipolarity at the level of an attribute).

Bipolarity can also be considered at the level of poles of criteria. In such a case multiple criteria are considered and subdivided into different poles of which the criteria have different semantics and require a different handling during criteria evaluation and aggregation. We refer to bipolarity that relates to multiple criteria as bipolarity that is specified among multiple criteria (cf. also [21], [23] where we refer to it as bipolarity at the level of the comprehensive evaluation). This kind of bipolarity is the subject of this paper. Different approaches to handling (aggregating) bipolar criteria has been proposed in the literature. Two of them are the constraint-wish approach and the satisfied-dissatisfied approach.

In the constraint-wish approach, the criteria in one pole are considered to be constraints, i.e., mandatory criteria, while the criteria in the other pole are rather wishes, i.e., desired criteria. This approach is used amongst others by Dubois and Prade [4], [5] and by Liétard et al. [1], [12]-[14], [18].

In the satisfied-dissatisfied approach, a pole of positive criteria, i.e., criteria that should be satisfied, and a pole of negative criteria which should be dissatisfied, are considered. This approach is used amongst others by De Tré et al. [3], [15], [16] and Zadrożny et al. [21]. Remark that often, negative conditions might be translated to constraints, while positive conditions might be seen as wishes.

In this paper, we study bipolarity among multiple criteria from the different perspective of criteria aggregation and aggregation structures. From this point of view, bipolarity is a concept of asymmetric or contrasting logic properties of two inputs, or two groups of inputs. We discuss different interpretations of bipolarity in aggregation structures and try to classify bipolar concepts in two fundamental groups:

- Bipolarity based on positive (desirable) and negative (undesirable) inputs.

- Bipolarity based on nonuniform input-dependent annihilators.

For both groups we propose canonical forms of aggregators. 
The remainder of the paper is organised as follows. In Section II some preliminaries on bipolar criteria are presented, explaining the two approaches that will be discussed in the paper in more detail. Section III deals with aggregators for poles of desirable and undesirable criteria. Aggregation in case of nonuniform input-dependent annihilators is discussed in Section IV. Finally, Section V states some conclusions.

\section{PRELIMINARIES}

Pioneering work in the area of heterogeneous bipolar criteria handling has been done by Lacroix and Lavency in [11], which seems to be the first non-fuzzy approach where a distinction has been made between mandatory and desired query criteria. As mentioned earlier, desired and mandatory criteria can be viewed as specifying positive and negative information, respectively. Indeed, the opposite of a mandatory criterion specifies what must be rejected and thus what is considered as being negative with respect to the query result, whereas desired criteria specify what is considered as being positive. In Lacroix and Lavency's approach the positive criteria are of a somehow lesser importance than negative ones but this cannot be represented by a simple importance weighting of these criteria. Later on, this idea has been further developed and adapted to be used in 'fuzzy' criterion specification. Existing research approaches on 'fuzzy' bipolar criteria handling can mainly be categorised into two groups, which can be denoted as the satisfied-dissatisfied approaches and the constraintwish approaches. Both categories of approaches are briefly introduced in the next two subsections.

\section{A. Satisfied-Dissatisfied Approaches}

In general, negative and positive criteria may be treated as equally important. Then, we are interested on how to aggregate them under such an assumption. A first assumption that can be made, is to consider a 'positive' pole $\left(C^{\text {pos }}\right)$ and a 'negative' pole $\left(C^{n e g}\right)$ of elementary criteria. The criteria of each pole are connected using logical connectives [3]. Hereby, only the conjunction operator $(\wedge)$, the disjunction operator $(\vee)$ and negation operator $(\neg)$ can be used. If no brackets are used to enforce priority, then negation has overall priority over conjunction and disjunction and conjunction has priority over disjunction.

The connected positive criteria form a logical expression that expresses what is permitted, whereas the connected negative criteria form another logical expression that expresses what is not permitted. What is neither explicitly permitted, nor forbidden is considered to be unspecified, which could, among others, be due to indifference or hesitation of the user with respect to what is permitted or not, or due to the inability of the user to specify all (un)permitted values within the criteria. This assumption reflects the heterogeneous bipolar characteristic of the approach.

In order to keep the heterogeneous characteristics, the approach in [3] proposes to evaluate the logical expressions corresponding with both poles independently of each other and to express criterion satisfaction using bipolar satisfaction degrees (BSDs). A BSD is a pair

$$
(s, d), s, d \in[0,1]
$$

of independent values $s$ and $d$ where $s$ is called the satisfaction degree and $d$ is called the dissatisfaction degree [15], [16] (cf. also the concept of evidence couple [17]).

BSDs can be aggregated in several ways. The standard logical operators for conjunction $(\wedge)$, disjunction $(\vee)$ and negation $(\neg)$ are respectively,

- $\left(s_{1}, d_{1}\right) \wedge\left(s_{2}, d_{2}\right)=\left(\min \left(s_{1}, s_{2}\right), \max \left(d_{1}, d_{2}\right)\right)$,

- $\left(s_{1}, d_{1}\right) \vee\left(s_{2}, d_{2}\right)=\left(\max \left(s_{1}, s_{2}\right), \min \left(d_{1}, d_{2}\right)\right)$,

- $\neg(s, d)=(d, s)$.

The independent evaluation of the two logical expressions corresponding to the criteria poles $C^{\text {pos }}$ and $C^{\text {neg }}$ yields a $\operatorname{BSD}(s, d)$. The satisfaction degree $s$ is the result of the evaluation of the logical expression of $C^{\text {pos }}$, whereas the dissatisfaction degree $d$ is the result of the evaluation of the logical expression of $C^{\text {neg }}$.

For the aggregation of the satisfaction degrees resulting from the evaluation of the elementary criteria in the logical expression of $C^{\text {pos }}$, the following rule set has been proposed in [3]:

- The conjunction of two satisfaction degrees $s_{1}, s_{2} \in[0,1]$ is defined by

$$
s_{1} \wedge s_{2}=\min \left(s_{1}, s_{2}\right) .
$$

- The disjunction of two satisfaction degrees $s_{1}, s_{2} \in[0,1]$ is defined by

$$
s_{1} \vee s_{2}=\max \left(s_{1}, s_{2}\right) \text {. }
$$

For the aggregation of the dissatisfaction degrees resulting from the evaluation of the elementary criteria in the logical expression of $C^{n e g}$, the following rule set has been proposed in [3]:

- Conjunction. The conjunction of two dissatisfaction degrees $d_{1}, d_{2} \in[0,1]$ is defined by

$$
d_{1} \wedge d_{2}=\max \left(d_{1}, d_{2}\right)
$$

- Disjunction. The disjunction of two dissatisfaction degrees $d_{1}, d_{2} \in[0,1]$ is defined by

$$
d_{1} \vee d_{2}=\min \left(d_{1}, d_{2}\right)
$$

Both rule sets have been constructed in accordance with the semantics of the standard aggregation operators for BSDs as given above. Besides minimum and maximum, alternative aggregation operators, based on other triangular norms and co-norms, can be used if a reinforcement effect is needed or desired. 


\section{B. Constraint-Wish Approaches}

An alternative kind of bipolar approach in fuzzy criterion specification is to consider two poles of criteria as mandatory criteria and desired criteria. Bipolarity is thus studied considering queries with preferences as in [11] and the operator and possibly is employed to join two types of conditions. Thus, pairs:

$$
(c, w), c, w \in[0,1]
$$

are now considered, where $c$ is the satisfaction degree of $a$ constraint, i.e., a required/mandatory condition, while $w$ is the satisfaction degree of $a$ wish, i.e., a desired condition which is, in general, not obligatory.

The pair $(c, w)$ may be seen as a special interpretation of the pair $(s, d)$ of (1), where $c=1-d$ and $w=s$, i.e., the negative criterion is treated as a complement of the mandatory condition and the positive criterion is treated as a facultative condition, in a sense. Lacroix and Lavency in [11] propose to use the 'and possibly' to aggregate $c$ and $w$, what effectively makes it possible to replace a pair $(c, w)$ with a single value (in [11] originally the set $\{0,1\}$ is used instead of the interval $[0,1])$ :

$$
\text { and possibly: } \begin{aligned}
:[0,1] \times[0,1] & \rightarrow[0,1] \\
(c, w) & \mapsto u
\end{aligned}
$$

The semantics of the 'and possibly' operator is represented by the following formula:

$$
c_{i} \text { and possibly } w_{i}=\min \left(c_{i}, P O S S \Rightarrow w_{i}\right)
$$

where:

$$
\text { POSS }=\max _{j} \min \left(c_{j}, w_{j}\right)
$$

and $j$ goes over all the objects under evaluation. Thus, the 'and possibly' operator is context-sensitive and relates the possibility of satisfying both conditions $c$ and $w$ to the actual existence of an object which satisfies both of them.

Lacroix and Lavency proposed the 'and possibly' operator in the classical logical context, i.e., $c$ and $w$ are binary values. This approach has been adapted to the fuzzy case in [20] via the use of fuzzy logic connectives, as it is already shown in (4). Fuzzy logical connectives may be interpreted in many different ways. In [22] various properties of the 'and possibly' operator are postulated and it is verified how particular interpretations of fuzzy logical connectives preserve them.

More recently,the semantics of mandatory and desired criteria have been formalised in a slightly different context by means of the so-called and if possible and or else fuzzy connectives [2].

1) 'And If Possible': With $c_{1}$ and $c_{2}$ being (elementary) criteria, the expression ' $c_{1}$ and if possible $c_{2}$ ' is used to express a weak, non symmetric conjunction in the sense that $c_{2}$ is less important than $c_{1}$. From another perspective $c_{1}$ can be considered as a basic criterion which should be satisfied, whereas the satisfaction of $c_{2}$ is only desired, for example because it is more demanding.
Let $\gamma_{c}$ be a fuzzy evaluation function for criterion $c$, which takes cases from a universe of discourse $U$ as arguments, i.e.,

$$
\begin{aligned}
\gamma_{c}: U & \rightarrow[0,1] \\
x & \mapsto \gamma_{c}(x) .
\end{aligned}
$$

This evaluation function evaluates the criterion $c$ for a case or value $x \in U$ and returns a number from the unit interval, where 0 denotes not satisfied at all and 1 represents fully satisfied.

In [2], the semantics of the connective 'and if possible' have been defined by the following axioms.

$$
\begin{array}{cl}
C_{1}: & \gamma_{c_{1} \text { and if possible } c_{2}}(x) \geq \min \left(\gamma_{c_{1}}(x), \gamma_{c_{2}}(x)\right) \\
C_{2}: & \gamma_{c_{1} \text { and if possible } c_{2}(x) \leq \gamma_{c_{1}}(x)} \\
C_{3}: \quad \exists c_{1}, c_{2}: \gamma_{c_{1}} \text { and if possible } c_{2}(x) \neq \gamma_{c_{2}} \text { and if possible } c_{1}(x) \\
C_{4}: \quad \gamma_{c_{1}}(x) \geq \gamma_{c_{1}^{\prime}}(x) \Rightarrow \\
\quad \gamma_{c_{1} \text { and if possible } c_{2}}(x) \geq \gamma_{c_{1}^{\prime} \text { and if possible } c_{2}}(x) \\
C_{5}: \quad \gamma_{c_{2}}(x) \geq \gamma_{c_{2}^{\prime}}(x) \Rightarrow \\
\quad \gamma_{c_{1} \text { and if possible } c_{2}}(x) \geq \gamma_{c_{1} \text { and if possible } c_{2}^{\prime}}(x) \\
C_{6}: \quad \gamma_{c_{1} \text { and if possible } c_{2}(x)=\gamma_{c_{1}} \text { and if possible }\left(c_{1} \text { and } c_{2}\right)}(x) .
\end{array}
$$

The following definition for $\gamma_{c_{1}}$ and if possible $c_{2}$, satisfying all of the above axioms, has been proposed:

$$
\begin{aligned}
& \gamma_{c_{1} \text { and if possible } c_{2}}(x)= \\
& \qquad \min \left(\gamma_{c_{1}}(x), k \gamma_{c_{1}}(x)+(1-k) \gamma_{c_{2}}(x)\right)
\end{aligned}
$$

where $k \in] 0,1[$.

The main difference between the 'and possibly' operator, defined by (4) and the 'And If Possible' operator defined above is the fact that the latter is truth-functional while the former is not. Both operators make the notion of 'possibility' operational but in a different way.

In Section IV we show that (6) is a special case of a more general class of aggregation operators introduced by Dujmović in the seventies of the past century.

2) 'Or Else': The expression ' $c_{1}$ or else $c_{2}$ ' is used to express a strong, non symmetric disjunction in the sense that $c_{2}$ is considered at a less important level as $c_{1}$ and therefore is not a full alternative for $c_{1}$. From a slightly different perspective $c_{1}$ can be considered as the most demanding criterion which preferably has to be satisfied, but if this is not possible, $c_{2}$ is still considered as an acceptable alternative.

In [2], the semantics of the connective 'or else' have been defined by the following axioms.

$$
\begin{array}{ll}
D_{1}: & \gamma_{c_{1} \text { or else } c_{2}}(x) \leq \max \left(\gamma_{c_{1}}(x), \gamma_{c_{2}}(x)\right) \\
D_{2}: & \gamma_{c_{1}} \text { or else } c_{2}(x) \geq \gamma_{c_{1}}(x) \\
D_{3}: & \exists c_{1}, c_{2}: \gamma_{c_{1}} \text { or else } c_{2}(x) \neq \gamma_{c_{2}} \text { or else } c_{1}(x) \\
D_{4}: & \gamma_{c_{1}}(x) \geq \gamma_{c_{1}^{\prime}}(x) \Rightarrow \gamma_{c_{1} \text { or else } c_{2}}(x) \geq \gamma_{c_{1}^{\prime} \text { or else } c_{2}}(x) \\
D_{5}: & \gamma_{c_{2}}(x) \geq \gamma_{c_{2}^{\prime}}(x) \Rightarrow \gamma_{c_{1} \text { or else } c_{2}}(x) \geq \gamma_{c_{1} \text { or else } c_{2}^{\prime}}(x) \\
D_{6}: & \gamma_{c_{1} \text { or else } c_{2}}(x)=\gamma_{c_{1} \text { or else }\left(c_{1} \text { or } c_{2}\right)}(x)
\end{array}
$$

The following definition for $\gamma_{c_{1}}$ or else $c_{2}$, satisfying all of the above axioms, has been proposed:

$$
\gamma_{c_{1} \text { or else } c_{2}}(t)=\max \left(\gamma_{c_{1}}(t), k \gamma_{c_{1}}(t)+(1-k) \gamma_{c_{2}}(t)\right)
$$

where $k \in] 0,1[$.

In Section IV we show that (7) is a special case of a more general class of aggregation operators introduce by Dujmović. 


\section{Bipolarity BASEd on Desirable AND UNDESIRABLE CRITERIA}

Both the satisfied-dissatisfied approach and the constraintwish approach can also be studied from the perspective of aggregation and aggregation structures. Such a study is the scientific contribution of this paper. In this section the satisfieddissatisfied approach is handled. The study of the constraintwish approach is detailed in Section IV. For both approaches, canonical forms of aggregators are presented.

\section{A. General Considerations}

Considering criteria evaluation in the context of database querying or decision support, we can observe that all evaluation problems consist of defining a set of elementary criteria for a group of selected attributes, and then evaluating their contribution to the overall (output) suitability $z$ of an evaluated system. Often each elementary criterion is defined for a single attribute. In some cases we can identify two groups of criteria: criteria expressing what is desirable and criteria expressing what is undesirable. For example, a home buyer may consider the proximity to job location to be a desirable attribute, and the proximity to a noisy or polluted airport to be an undesirable attribute. Similarly a car buyer can consider the strong power of engine to be a desirable attribute, and the high cost of fuel and maintenance to be undesirable attributes. Infrequently the same criterion can be used to express what is desirable (from some standpoint or in a given interval of values) and what is undesirable (from another standpoint, or in another interval of values). In such rare cases the same criterion may be a member of both groups. In what follows, we will briefly call an attribute desirable (resp. undesirable) if its corresponding criterion expresses what is desirable (resp. undesirable).

For the sake of simplicity, assume that elementary criteria may be expressed only on the level of individual attributes. With the understanding that $a$ denotes an attribute, dom ${ }_{a}$ denotes its associated domain of allowed values and $[a]$ denotes the actual value of $a$ under consideration, a desirable and an undesirable group of attributes can be specified as follows.

The desirable group of criteria includes $n$ elementary criteria $g_{i}: \operatorname{dom}_{a_{i}} \rightarrow I=[0,1], i=1, \ldots, n$ for $n$ attributes $a_{1}, \ldots, a_{n}$, hereafter called the desirable attributes, that are evaluated so that the attribute suitability degrees $x_{i}=g_{i}\left(\left[a_{i}\right]\right)$ satisfy the condition $\partial z / \partial x_{i} \geq 0, i=1, \ldots, n$, with $z$ being the overall, aggregated (output) suitability.

The undesirable group of criteria includes $k$ elementary criteria $h_{i}:$ dom $_{u_{i}} \rightarrow I, i=1, \ldots, k$ for $k$ attributes $u_{1}, \ldots, u_{k}$, hereafter called the undesirable attributes, that are evaluated so that the attribute unsuitability degrees $y_{i}=h_{i}\left(\left[u_{i}\right]\right)$ satisfy the condition $\partial z / \partial y_{i} \leq 0, i=1, \ldots, k$.

\section{B. Canonical Aggregation Structures}

In the area of logic aggregation structures some structures have an easily justifiable regular form. Such structures are called canonical structures. A survey of frequently used

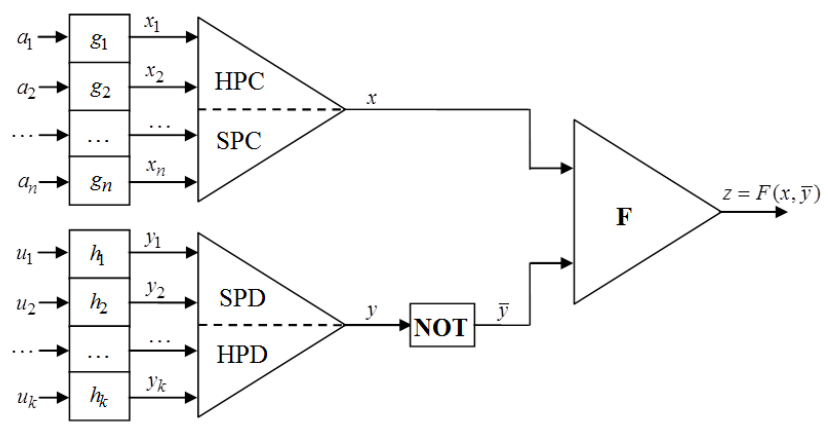

Fig. 1. The structure of canonical LSP bipolar criterion.

canonical aggregation structures can be found in [10]. Bipolar criteria with the desirable/undesirable attributes can be modelled using a bipolar canonical LSP criterion structure shown in Figure 1. Logic Scoring of Preference (LSP) is a decision support technique for the specification, evaluation and aggregation of multiple criteria [7], [9]. Aggregation in LSP is done by using an aggregation structure. This structure is specifically designed for the decision process under consideration and has to reflect the human decision making process as adequate as possible. The basic components of the aggregation structure are the simple LSP aggregators, which act as logical connectives. Simple LSP aggregators can on their turn be combined into compound aggregators.

1) LSP Aggregators: The formal basis for LSP aggregators is the so-called generalised conjunction/disjunction (GCD) function which can be expressed by

$$
\begin{aligned}
& M\left(x_{1}, \ldots, x_{n} ; W_{1}, \ldots, W_{n} ; r\right)= \\
& \begin{cases}\left(\sum_{i=1}^{n} W_{i} x_{i}^{r}\right)^{1 / r} & , \text { if } 0<|r|<+\infty \\
\prod_{i=1}^{n} x_{i}^{W_{i}} & , \text { if } r=0 \\
x_{1} \wedge \cdots \wedge x_{n} & , \text { if } r=-\infty \\
x_{1} \vee \cdots \vee x_{n} & , \text { if } r=+\infty\end{cases}
\end{aligned}
$$

where the values $x_{i} \in[0,1], 1 \leq i \leq n$ are the input suitability degrees (hereby, 0 and 1 respectively denote 'not suitable at all' and 'completely suitable'); the given (or precomputed) weights $W_{i}, 1 \leq i \leq n$ determine the relative importance of the inputs and have to sum up to 1 ; and the given (or precomputed) exponent $r \in[-\infty,+\infty]$ determines the logical properties of the aggregator. Special cases of exponent values are: $+\infty$ corresponding to full disjunction, $-\infty$ corresponding to full conjunction, and 1 corresponding to weighted average. The other exponent values allow to model other aggregators, ranging continuously from full conjunction to full disjunction and can be computed from a desired value of orness $(\omega)$, i.e., an index expressing how 'close' the aggregator should be in its behavior to the regular disjunction operator. The following numeric approximation for $r$ can be used [9]:

$$
r=\frac{0.25+1.89425 x+1.7044 x^{2}+1.47532 x^{3}-1.42532 x^{4}}{\omega(1-\omega)}
$$


where

$$
x=\omega-1 / 2 \text { and } 0<\omega<1 .
$$

The andness $(\alpha)$ is obtained as the complement of the orness, i.e.,

$$
\alpha=1-\omega .
$$

Andness is hence an index expressing how 'close' the aggregator should be in its behavior to the regular conjunction operator.

For $\omega>0.5$ we have disjunction, $\omega=1$ corresponds with $r=+\infty$ and is called full disjunction. For $0.75<\omega<1$ a hard partial disjunction (HPD) operator is obtained, whereas $0.5<\omega<0.75$ yields a soft partial disjunction (SPD) operator. So, $\omega=0.75$ can be considered as corresponding with a neutral partial disjunction operator.

Likewise, for $\alpha>0.5$ we have conjunction, $\alpha=1$ corresponds with $r=-\infty$ and is called full conjunction. For $0.75<\alpha<1$ a hard partial conjunction (HPC) operator is obtained, whereas $0.5<\alpha<0.75$ yields a soft partial conjunction (SPC) operator. Also here, $\alpha=0.75$ can be considered as corresponding with a neutral partial conjunction operator.

If $\alpha=\omega=0.5$ the neutral (weighted) arithmetic mean operator is obtained. This corresponds with the case where $r=1$.

2) Aggregators for Desirable and Undesirable Criteria: Now, reconsider the canonical LSP criterion structure shown in Figure 1. For the group of desirable attributes $a_{1}, \ldots, a_{n}$, we have that the higher their corresponding suitability degrees are, the better the situation is. We now assume that this group of attributes can be separated in two subgroups: mandatory and non-mandatory attributes. Mandatory attributes are aggregated using an aggregation structure based on HPC. By definition, HPC is an aggregator that has the annihilator 0 supported (uniformly) by all inputs, i.e., if any of the input suitability degrees is 0 , then the output must also be 0 . Non-mandatory desired attributes are aggregated by an aggregation structure that is based on SPC. SPC is a conjunctive aggregator that does not support the annihilator 0; only one positive input is sufficient to secure a positive output. If all mandatory inputs are aggregated using a HPC structure that yields the suitability $x_{m a n}$ and all nonmandatory (desired or optional) inputs are aggregated using a SPC structure that yields the suitability $x_{n m a n}$, then the aggregated suitability $x$ can be obtained by using a conjunctive partial absorption (CPA) operator $\unrhd[8]$, i.e., $x=x_{\text {man }} \unrhd x_{n \text { man }}$. CPA is further discussed in the next section.

For the group of undesirable attributes $u_{1}, \ldots, u_{k}$, we have that the higher their corresponding suitability degrees are, the worse the situation is. We can separate this group of attributes in two groups: sufficient and non-sufficient attributes. Sufficient undesired attributes are aggregated using an aggregation structure based on HPD. By definition, HPD is an aggregator that has the annihilator 1 supported (uniformly) by all inputs, i.e., if any of the input unsuitability degrees is 1 , then the output must also be 1 . This makes each input in such a group sufficient to point out that the evaluated object is unacceptable. Non-sufficient undesired attributes are aggregated by an aggregation structure that is based on SPD. SPD is a disjunctive aggregator that does not support the annihilator 1; only one input that is less than 1 is sufficient to result in output that is less than 1 . If all sufficient undesired inputs are aggregated using a HPD structure that yields the unsuitability $y_{s u f}$ and all nonsufficient undesired inputs are aggregated using a SPD structure that yields the unsuitability $y_{n s u f}$, then the aggregated unsuitability $y$ can be aggregated using a disjunctive partial absorption (DPA) operator $\bar{\triangleright}[8]$, i.e., $y=y_{s u f} \triangleright y_{n s u f}$. DPA is further discussed in the next section.

The aggregation of the desirable attribute scores (suitability degrees) generates the overall suitability score $x$, whereas the aggregation of the undesirable attribute scores (unsuitability degrees) generates the overall unsuitability degree $y$ as shown in Figure 1. The degrees $x$ and $y$ have similar semantics as the satisfaction degree $s$ and dissatisfaction degree $d$ of the BSD $(s, d)$ that is obtained in the satisfied-dissatisfied approach presented in [3] (cf. Eq. 1).

However in the canonical aggregation structure given in Figure 1 it is proposed to aggregate $x$ and $y$ in order to obtain an overall suitability degree $z$. Let us now consider a process of aggregating the suitability $x$ and the unsuitability $y$. A simplified verbal criterion could be 'we simultaneously want $x$ and not $y$.' In an extreme Boolean case that yields the material nonimplication or the abjunction function $z=x \wedge \bar{y}$. The abjunction function is an nonidempotent aggregator which can also be denoted as a negated implication: $x \wedge \bar{y}=\overline{x \rightarrow y}$.

Not surprisingly, in a general case we can apply a partial abjunction function based on GCD. To convert the unsuitability score $y$ to a corresponding suitability score $\bar{y}$ we need negation, i.e.,

$$
\bar{y}=(1-y)^{p}
$$

where the parameter $p$ is frequently selected to be 1 , but in some cases it can be greater than 1 or less than 1 .

The final stage of the canonical aggregation structure is the aggregation of suitability of desirable attributes $x$ and unsuitability of undesirable attributes $y$ creating the output overall suitability

$$
z=F(x, \bar{y}) .
$$

The output aggregation function $F$ can take several forms, not necessarily referring to the strict abjunction mentioned above. In particular, $F$ can be a HPC operator, i.e., $z=W x \triangle \bar{W} \bar{y}$, $0<W<1, \bar{W}=1-W, \bar{y}=y-1$. This aggregator is a weighted partial abjunction of $x$ and $y$. In some cases it can be justifiable to use SPC. On the other hand, a special interpretation of desirable and undesirable attributes may be assumed, e.g., the one related to the constraint-wish approach. Then, $F$ can be an asymmetric aggregator, where $x$ is a mandatory input and $\bar{y}$ is an optional input (i.e., a CPA [8]).

The canonical aggregation structure from Figure 1 can be transformed if the elementary criteria of undesired attributes can be 'negated' as exemplified in Figure 2. In this example if 

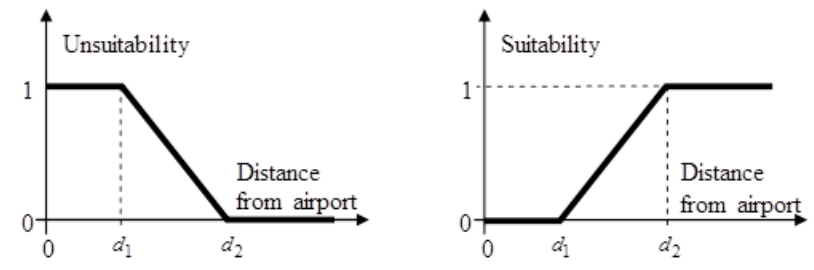

Fig. 2. Suitability and unsuitability criteria for the distance of home from an airport.

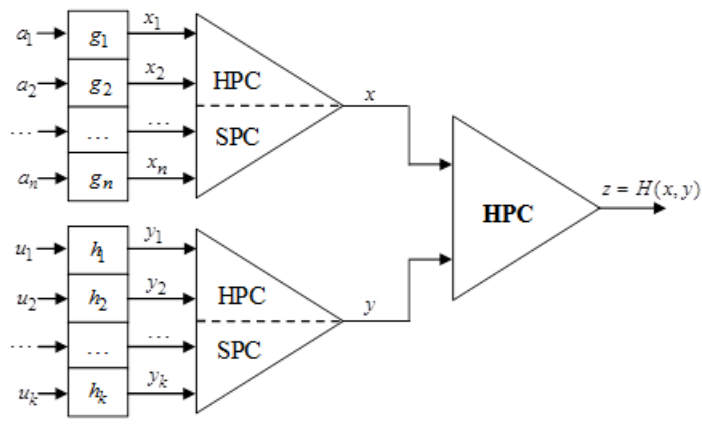

Fig. 3. Symmetric canonical aggregation structure.

the distance from the airport is undesirable, then it is equivalent to use either the unsuitability criterion or its complement, the suitability criterion.

If all undesired attributes are evaluated using suitability criteria, then we can replace the nonidempotent canonical aggregation structure from Figure 1 with the idempotent canonical aggregation structure shown in Figure 3. It is useful to note that the canonical aggregation structures in both Figure 1 and Figure 3 use 'bipolarity inside bipolarity' (or 'multipolarity') identifying four categories of attributes: (1) mandatory desired, (2) optional desired, (3) mandatory undesired, and (4) optional undesired. Thus, bipolarity, tripolarity [19] (mandatory/desired/optional, shown in Figure 4), and generally 'multipolarity' reflect situations where we have two or more logically dissimilar clusters of attributes and each cluster contains attributes that have a similar logical impact on the overall suitability score.

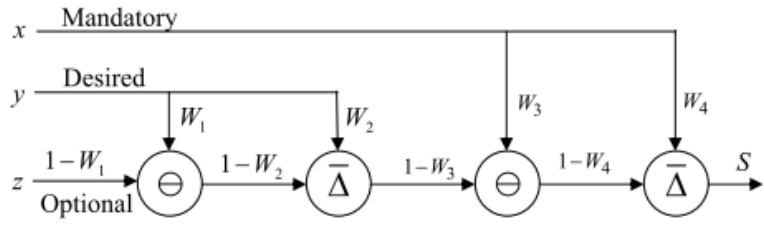

Fig. 4. Tripolarity realized using nested bipolarity: $S=x \unrhd(y \unrhd z)$.
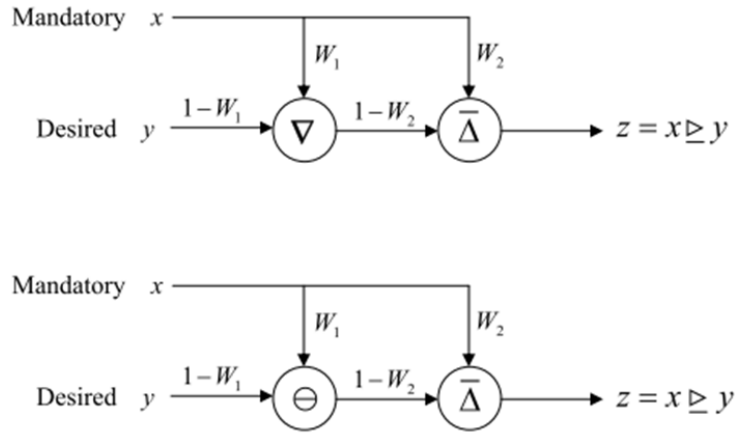

Fig. 5. Two basic versions of conjunctive partial absorption.
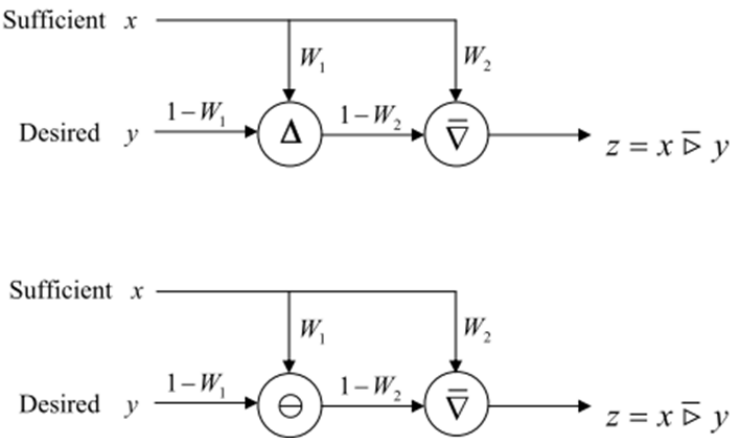

Fig. 6. Two basic versions of disjunctive partial absorption.

\section{BIPOLARITY BASED ON NONUNIFORM INPUT-DEPENDENT ANNIHILATORS}

\section{A. General Considerations}

The interpretations of constraint-wish approaches of bipolarity are based on asymmetric aggregation operators. Among them, the Conjunctive Partial Absorption $\unrhd$ (CPA) and the Disjunctive Partial Absorption $\bar{\triangleright}$ (DPA) seem especially interesting. The concept of asymmetric aggregators and the CPA can be considered as the oldest and perhaps most frequently used form of bipolarity introduced in 1974 [6]. Both the CPA and the DPA were then studied in [7]. A detailed quantitative analysis and synthesis of PA aggregators were presented in [8].

\section{B. Canonical Aggregation Structures}

The basic canonical forms of CPA and DPA aggregators have two inputs: $x$ and $y$ and their internal organization is presented in Figure 5 and Figure 6.

In these figures the symbols $\nabla, \Delta$ and $\ominus$ respectively denote partial disjunction, partial conjunction, and the neutrality (arithmetic mean). More specifically, $\bar{\nabla}$ denotes HPD and $\bar{\Delta}$ denotes HPC, whereas $W_{1}$ and $W_{2}$ are precomputed weights (see below).

In the case of CPA $\unrhd$ (Figure 5) the input $x$ is mandatory and it supports the annihilator 0, i.e.,

$$
\forall y>0: 0 \unrhd y=0 .
$$


As opposed to that, the optional input $y$ does not support the annihilator 0, i.e.,

$$
\forall x>0: 0<x \unrhd 0<x .
$$

Similarly, in the case of DPA $\bar{\triangleright}$ (Figure 6) the input $x$ is sufficient and it supports the annihilator 1, i.e.,

$$
\forall y<1: 1 \bar{\triangleright} y=1 \text {. }
$$

As opposed to that, the optional input $y$ does not support the annihilator 1, i.e.,

$$
\forall x<1: x<x \unrhd 1<1 .
$$

The CPA $\unrhd$ (Figure 5) aggregates a mandatory input $x$ and a non-mandatory (desired or optional) input $y$, as follows:

$$
x \unrhd y=W_{2} x \overline{\bar{\Delta}}\left(1-W_{2}\right)\left[W_{1} x \tilde{\nabla}\left(1-W_{1}\right) y\right]
$$

where $\overline{\bar{\Delta}} \in\{\wedge, \bar{\Delta}\}$ and $\tilde{\nabla} \in\{\vee, \underline{\nabla}, \bar{\nabla}, \ominus\}$. Here, $\bar{\Delta}$ denotes hard partial conjunction, $\underline{\nabla}$ represents soft partial disjunction, and $\bar{\nabla}$ denotes hard partial disjunction.

The DPA $\bar{\nabla}$ (Figure 6) aggregates a sufficient input $x$ and a non-sufficient (desired or optional) input $y$, as follows:

$$
x \bar{\triangleright} y=W_{2} x \overline{\bar{\nabla}}\left(1-W_{2}\right)\left[W_{1} x \tilde{\Delta}\left(1-W_{1}\right) y\right]
$$

where $\overline{\bar{\nabla}} \in\{\vee, \bar{\nabla}\}$ and $\tilde{\Delta} \in\{\wedge, \underline{\Delta}, \bar{\Delta}, \ominus\}$. Here, $\bar{\nabla}$ denotes hard partial disjunction, $\Delta$ represents soft partial conjunction and, $\bar{\Delta}$ denotes the hard partial conjunction.

In both equations Eq. (12) and (13), the weights $W_{1}$ and $W_{2}$ are computed so as to reflect as adequately as possible the impact of the mean penalty $P$ and mean reward $R$ percentages provided by the user. Hereby the underlying semantics of $P$ and $R$ are defined by the following border conditions for the $\mathrm{CPA} \unrhd[8]$ (and their dual counterparts for the DPA $\bar{\triangleright}$ ):

$$
\forall 0<x \leq 1: x \unrhd 0=x(1-p), 0 \leq p<1
$$

(hence if the optional condition is not satisfied at all, then criterion satisfaction is decreased with a penalty of $p$ )

$$
\forall 0<x<1: x \unrhd 1=x(1+r), 0 \leq r<1 / x-1
$$

(hence if the optional condition is fully satisfied, then criterion satisfaction is increased with a reward of $r$ ). Note that $p$ and $r$ can be zero. The values $P$ and $R$ are (approximately) the mean values of $p$ and $r$ and usually expressed as percentages. Users select desired values of $P$ and $R$ and use them to compute the corresponding weights $W_{1}$ and $W_{2}$. More details on this computation can be found in [8].

Let us note that Eq. (6) is a special case of Eq. (12) that is obtained if $\overline{\bar{\Delta}}=\wedge$ and $\tilde{\nabla}=\ominus$. The use of (hard) partial conjunction in Eq. (12) enables the use of a reward. It can also be seen that Eq. (7) is a special case of Eq. (13), which is obtained if $\bar{\nabla}=\vee$ and $\tilde{\Delta}=\ominus$. The use of (hard) partial disjunction in Eq. (13) enables the use of a penalty.

\section{Comparison with the 'And if possible' and 'Or else' Operators}

Studying the fundamental properties of CPA and DPA and comparing them with those of the 'and if possible' and 'or else' operators presented in Section II we obtain the following.

The fundamental properties of CPA are:

1) $\forall 0 \leq y \leq 1: 0 \unrhd y=0$

2) $\forall 0<x \leq 1: 0<x \unrhd 0 \leq x$

3) $\forall 0<x<1: x \leq x \unrhd 1<1$

Comparing these properties with the axioms of the 'and if possible' operator reveals that axiom $\left[\mathrm{C}_{2}\right]$ is in conflict with the third fundamental property of CPA. In fact, axiom $\left[\mathrm{C}_{2}\right]$ permits penalising and prevents rewarding. For example, in a criterion for quality of available parking facilities at a home, let the mandatory requirement be the availability of a private garage for one or ideally for two cars. Let the optional requirement be the availability of a quality street parking. Now, if the mandatory requirement is partially or perfectly satisfied, but the street parking is not available, the function (6) will penalise such a home. However, if a mandatory requirement is partially satisfied (there is a garage for one car), and there is a perfect street parking, the function (6) does not permit to compensate imperfections of the home parking with the quality street parking, and this may be inconsistent with human reasoning.

The fundamental properties of DPA are:

1) $\forall 0 \leq y \leq 1: 1 \bar{\triangleright} y=1$

2) $\forall 0<x<1: 0<x \bar{\triangleright} 0 \leq x$

3) $\forall 0<x<1: x \leq x \bar{\triangleright} 1<1$

Comparing these properties with the axioms of the 'or else' operator reveals that axiom $\left[D_{2}\right]$ is in conflict with the third fundamental property of DPA. In fact, based on axiom $\left[D_{2}\right]$, the aggregator (7) permits only rewards and no penalty, which is frequently inconsistent with human reasoning. Consider for example two car descriptions where a sufficient criterion 'low fuel consumption' is perfectly satisfied for both. If a desired condition 'airbags' is only satisfied for the first car and there is no penalty facility available, then it would not be possible to distinguish between the overall satisfaction of both cars. However, humans would naturally assign a penalty to the second car and prefer the first one.

The main advantage of the CPA and DPA operators is that they enable the use of both a reward and a penalty. As illustrated above, both rewards and penalties are required if we want to adequately reflect human reasoning.

\section{Conclusions}

In this paper we discussed and studied bipolarity among multiple criteria from the standpoint of aggregation and aggregation structures. Two main approaches are distinguished: bipolarity based on desirable and undesirable criteria and bipolarity based on nonuniform input-independent annihilators. For both approaches canonical forms of aggregators are proposed. Bipolarity based on desirable and undesirable criteria is approached by considering different poles of criteria 
which all have their own different logic semantics. Herewith it is illustrated how bipolarity, so-called tripolarity and more general multipolarity (or 'multi-level bipolarity') can be considered. Bipolarity based on nonuniform input-dependent annihilators is studied from the standpoint of conjunctive and disjunctive partial absorption. Herewith it is shown that the 'and possibly' and 'or else' operators of the well known 'constraint-wish' approach, are in fact special cases of partial absorption. In our future research, we plan to further explore the concepts of tripolarity and multipolarity.

\section{REFERENCES}

[1] Bosc, P., Pivert, O., Mokhtari, AM, Liétard, L.: Extending relational algebra to handle bipolarity. In: Proc. of SAC'10, Sierre, Switzerland, pp. 1718-1722 (2010).

[2] Bosc, P., Pivert, O.: On four noncommutative fuzzy connectives and their axiomatization. Fuzzy Sets and Systems 202, 42-60 (2012).

[3] De Tré, G., Zadrożny, S., Matthé, T., Kacprzyk, J., Bronselaer, A.: Dealing with positive and negative query criteria in fuzzy database querying : bipolar satisfaction degrees. Lecture Notes in Computer Science 5822, 593-604 (2009).

[4] Dubois, D., Prade, H.: Handling bipolar queries in Fuzzy Information Processing. In: Galindo J. (ed.) Handbook of Research on Fuzzy Information Processing in Databases, IGI Global, New York, USA, pp. 97-114 (2008).

[5] Dubois, D., Prade, H.: An introduction to bipolar representations of information and preference. International Journal of Intelligent Systems 23, 866-877 (2008).

[6] Dujmović, J.J.: New Results in the Development of the Mixed Averaging By Levels Method for System Evaluation (In Serbo-Croatian). In: Proc. of the Informatica Conference, Bled, Yugoslavia, paper 4.36 (1974).

[7] Dujmović, J.J.: Extended Continuous Logic and the Theory of Complex Criteria. Journal of the University of Belgrade 537, 197-216 (1975).

[8] Dujmović, J.J.: Partial Absorption Function. Journal of the University of Belgrade, 659, 156-163 (1979).

[9] Dujmović, J.J.: Preference Logic for System Evaluation. IEEE Trans. on Fuzzy Systems 15(6), 1082-1099 (2007).

[10] Dujmović, J.J., De Tré, G.: Multicriteria Methods and Logic Aggregation in Suitability Maps. International Journal of Intelligent Systems 26(10), 971-1001 (2011).

[11] Lacroix, M., Lavency, P.: Preferences: Putting more knowledge into queries. In: Proc. of the VLDB'87 Conference, Brighton, UK, pp. 217-225 (1987).

[12] Liétard, L., Rocacher, D.: On the Definition of Extended Norms and Co-norms to Aggregate Fuzzy Bipolar Conditions. In: Proc. of the 2009 IFSA/EUSFLAT Conference, Lisbon, Portugal, pp. 513-518 (2009).

[13] Liétard, L., Tamani, N., Rocacher, D.: Linguistic Quantifiers and Bipolarity. In: Proc. of the 2011 IFSA World Congress and the 2011 AFSS International Conference, Surabaya and Bali Island, Indonesia (2011).

[14] Liétard, L., Pivert, O., Rocacher, D.: On a graded inclusion of bipolar fuzzy relations. In: Proc. of the 8th EUSFLAT Conference, Milan, Italy (2013).

[15] Matthé, T., De Tré, G.: Bipolar query satisfaction using satisfaction and dissatisfaction degrees: bipolar satisfaction degrees. In: Proc. of the ACM Symposium on Applied Computing (ACM SAC'09), Honolulu, Hawaii, pp. 1699-1703 (2009).

[16] Matthé, T., De Tré, G., Zadrożny, S., Kacprzyk, J., Bronselaer, A.: Bipolar database querying using bipolar satisfaction degrees. International Journal of Intelligent Systems 26(10), 890-910 (2011).

[17] Rodriíguez, J.T., Camilo, A.F., Montero F.J.: On the Semantics of Bipolarity and Fuzziness. In: Melo-Pinto P. et al. (eds.) Eurofuse 2011: Workshop on Fuzzy Methods for Knowledge-Based Systems, Springer, Heidelberg, Germany, pp. 193-205 (2012).

[18] Tamani, N., Liétard, L., Rocacher, D.: Bipolarity and the relational division. In: Proc. of the 7th EUSFLAT Conference, Aix-les-Bains, France (2011).

[19] Su, S.Y.W., Dujmović, J.J., Batory, D.S., Navathe, S.B., Elnicki, R.: A Cost-Benefit Decision Model: Analysis, Comparison, and Selection of Data Management Systems. ACM Transactions on Database Systems 12(3), 472-520 (1987)
[20] Zadrożny, S., Kacprzyk, J.: Bipolar queries and queries with preferences. In:Proc. of the DEXA Conference (DEXA'06), Krakow, Poland, pp. 415419 (2006).

[21] Zadrożny, S., De Tré, G., Kacprzyk, J.: Remarks on various aspects of bipolarity in database querying. In: Proc. of the 2010 International Workshop on Database and Expert Systems Applications Proceedings (DEXA'10), Bilbao, Spain, pp. 323-327 (2010).

[22] Zadrożny, S., Kacprzyk, J.: Bipolar queries: An aggregation operator focused perspective. Fuzzy Sets and Systems 196, 69-81 (2012).

[23] Zadrożny, S., Kacprzyk, J.: Bipolarity in Database Querying: Various Aspects and Interpretations. In: Pivert, O., Zadrożny, S. (eds.) Flexible Approaches in Data, Information and Knowledge Management, Springer, Heidelberg, Germany, pp. 71-91 (2013). 